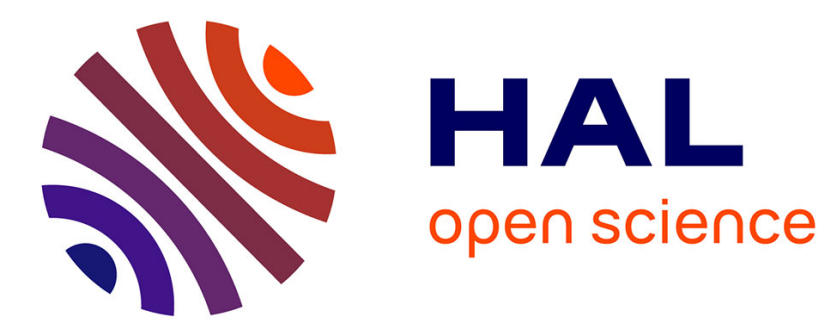

\title{
A Closed-Form Solution of Rotation Invariant Spherical Harmonic Features in Diffusion MRI
}

\author{
Mauro Zucchelli, Samuel Deslauriers-Gauthier, Rachid Deriche
}

\section{To cite this version:}

Mauro Zucchelli, Samuel Deslauriers-Gauthier, Rachid Deriche. A Closed-Form Solution of Rotation Invariant Spherical Harmonic Features in Diffusion MRI. MICCAI - Computational Diffusion MRI Workshop 2018, Sep 2018, Granada, Spain. hal-01912148

\author{
HAL Id: hal-01912148 \\ https://hal.inria.fr/hal-01912148
}

Submitted on 5 Nov 2018

HAL is a multi-disciplinary open access archive for the deposit and dissemination of scientific research documents, whether they are published or not. The documents may come from teaching and research institutions in France or abroad, or from public or private research centers.
L'archive ouverte pluridisciplinaire HAL, est destinée au dépôt et à la diffusion de documents scientifiques de niveau recherche, publiés ou non, émanant des établissements d'enseignement et de recherche français ou étrangers, des laboratoires publics ou privés. 


\title{
A Closed-Form Solution of Rotation Invariant Spherical Harmonic Features in Diffusion MRI
}

\author{
Mauro Zucchelli, Samuel Deslauriers-Gauthier, and Rachid Deriche \\ Athena Project-Team, Inria Sophia Antipolis - Méditerranée, France
}

\begin{abstract}
Rotation invariant features are an indispensable tool for characterizing diffusion Magnetic Resonance Imaging (MRI) and in particular for brain tissue microstructure estimation. In this work, we propose a new mathematical framework for efficiently calculating a complete set of such invariants from any spherical function. Specifically, our method is based on the spherical harmonics series expansion of a given function of any order and can be applied directly to the resulting coefficients by performing a simple integral operation analytically. This enable us to derive a general closed-form equation for the invariants. We test our invariants on the diffusion MRI fiber orientation distribution function obtained from the diffusion signal both in-vivo and in synthetic data. Results show how it is possible to use these invariants for characterizing the white matter using a small but complete set of features.
\end{abstract}

Keywords: Diffusion MRI, Rotation Invariant, Gaunt Coefficients, Spherical Harmonics

\section{Introduction}

Brain tissue microstructure influences the diffusion Magnetic Resonance Imaging (MRI) signal in-vivo in multiple ways. Tissue microstructure in gray and white matter can be subdivided into two main categories: tissue composition and tissue orientation. Tissue composition corresponds to the heterogeneity of the neurites in the voxel, namely the presence and relative fraction of a certain neurite population in the voxel. Axonal diameter and intra-axonal signal fraction are two of the most studied tissue composition microstructural features in diffusion MRI. Tissue orientation, on the other hand, corresponds to the geometrical arrangement of the neurites in a given voxel. White matter axonal bundles (also known as "fiber bundles"), because of their high anisotropy, represent the key factor which influences the diffusion MRI signal via tissue orientation. It is common practice in diffusion MRI to model the tissue orientation information in each voxel using the so-called fiber Orientation Distribution Function (fODF) [18]. The fODF represents the probability distribution function of the fibers, namely the probability of having a fiber oriented in a given direction. Such a distribution is commonly obtained from the diffusion signal by deconvolving it with a single fiber response which, in theory, should correspond to the tissue composition in 
the same location [3][18][21]. In diffusion MRI, fODF is modeled using its real symmetric Spherical Harmonics (SH) series expansion, and therefore, each fODF is completely characterized by its $\mathrm{SH}$ coefficients. Being a well defined numerical quantity, the idea of using the SH coefficients directly as a biomarker for tissue orientation is appealing but it suffers from the fact that even a small rotation of the same fODF will produce a different set of coefficients, which makes their use unsuitable for classification and clustering tasks. An intuitive solution to this problem is to use the SH coefficients to calculate Rotation Invariant Features (RIF) which, as the name implies, have values which are independent of the direction of the fODF. The first and most well-known fODF RIF which has been developed for diffusion MRI is the Generalized Fractional Anisotropy (GFA) [19]. GFA represents the fODF evolution of the diffusion tensor-derived Fractional Anisotropy (FA) with the advantage of being able to better represent crossing fibers. However, reducing the rich directional information present in the fODF into a single numerical value severely limits its descriptive power. Bloy and Verma [1] proposed to use the power spectrum of the SH coefficients to register diffusion MR images. The power spectrum provides a richer description with respect to GFA but is still incomplete with respect to the theoretical number of independent invariants obtainable. Ghosh et al. [6] [13] proposed a method for recovering all 12 invariants obtainable from a $4^{\text {th }}$ order tensor while Caruyer and Verma [2] derived a framework for obtaining all the algebraically independent invariants for any given order of SH. Such a framework initially derives all the polynomials invariant to a rotation of 1 degree with respect to the $x$ and $z$ axes, which are proven to be invariant to any additional rotation. After collecting all the possible invariants, a pruning algorithm is applied to the polynomials to reduce them to the minimal set of algebraically independent invariants. The main limitations of this technique is that the invariants cannot be expressed in closed-form and that it produces a huge number of polynomials to be tested for independence (more than 30,000 for a SH order of 6).

The goal of this work is to propose a new framework for generating a set of invariants in closed-form (Section 2) with an elegant mathematical framework. Our solution is able to produce fewer polynomials to be tested for independence compared to previous published related work. In Section 3 we will present the synthetic and in-vivo fODF data which we will use to test our RIF. Results show the potential for these invariants to disambiguate complex fiber geometries using only a small set of invariants (Section 4).

\section{Theory}

\subsection{A new set of invariants for $\mathrm{SH}$}

Given a spherical function $f(\mathbf{u})$ defined on the three dimensional unit vector $\mathbf{u}$ we can represent it as a linear combination of $\mathrm{SH}$ as

$$
f(\mathbf{u})=\sum_{l=0}^{\infty} \sum_{m=-l}^{l} c_{l m} Y_{l}^{m}(\mathbf{u})
$$


where $Y_{l}^{m}$ are the real SH functions [4][7], and $c_{l m} \in \mathbb{R}$ are the SH coefficients. The coefficients $c_{l m}$ are calculated as the inner product $f$ and $Y_{l}^{m}$

$$
c_{l m}=\int_{S^{2}} f(\mathbf{u}) Y_{l}^{m}(\mathbf{u}) d \mathbf{u} .
$$

A rotation $R$ applied to the $\mathrm{SH}$ functions can be written as

$$
R Y_{l}^{m}(\mathbf{u})=Y_{l}^{m}\left(R^{-1} \mathbf{u}\right)=\sum_{m^{\prime}=-l}^{l} D_{m^{\prime}, m}^{l}(R) Y_{l}^{m^{\prime}}(\mathbf{u})
$$

with $D_{m^{\prime}, m}^{l}(R)$ defined as

$$
D_{m^{\prime}, m}^{l}(R)=e^{-i m^{\prime} \alpha} d_{m^{\prime}, m}^{l}(\beta) e^{-i m \gamma}
$$

where $\alpha, \beta$, and $\gamma$ are the Euler angles and $d_{m^{\prime}, m}$ is the Wigner $d$ function. Therefore, we can define $h(\mathbf{u})=R f(\mathbf{u})$ the function $f$ rotated by $R$ and whose $\mathrm{SH}$ expansion is given by

$$
\begin{aligned}
h(\mathbf{u}) & =R f(\mathbf{u})=\sum_{l=0}^{\infty} \sum_{m=-l}^{l} c_{l m} Y_{l}^{m}\left(R^{-1} \mathbf{u}\right) \\
& =\sum_{l=0}^{\infty} \sum_{m^{\prime}=-l}^{l} g_{l m^{\prime}} Y_{l}^{m^{\prime}}(\mathbf{u}) .
\end{aligned}
$$

The rotated SH coefficients for a given $l$ are given by

$$
g_{l m^{\prime}}=\sum_{m=-l}^{l} c_{l m} D_{m^{\prime}, m}^{l}(R) .
$$

A RIF of a Spherical Function $f$ can be expressed as a function $I$ for which

$$
I[f(\mathbf{u})]=I[R f(\mathbf{u})], \forall R
$$

If $f$ is expressed in terms of its $\mathrm{SH}$ expansion, we have that

$$
I[\mathbf{c}]=I[\mathbf{g}]
$$

where $\mathbf{c}$ and $\mathbf{g}$ are the SH coefficients of $f(\mathbf{u})$ and $h(\mathbf{u})$, respectively. It is straightforward to see that the integral of $f(\mathbf{u})$ represents a RIF, in fact:

$$
I[f]=\int_{S^{2}} f(\mathbf{u}) d \mathbf{u}=\int_{S^{2}} f\left(R^{-1} \mathbf{u}\right) d \mathbf{u}
$$

this equivalence follows the natural law that the area of a function does not change while rotating the function. In particular in this case $I[f]=\int_{S^{2}} f(\mathbf{u}) d \mathbf{u}=$ $c_{00} \sqrt{4 \pi}$, and therefore, normalizing by the constant factor $\sqrt{4 \pi}$, the polynomial 
$P(\mathbf{c})=c_{00}$ represents a first RIF for $f$. Similarly, we can prove that if we raise the function $f$ to the power $d$, the integral of such a function is still a RIF. In fact

$$
I^{d}[f]=\int_{S^{2}}[f(\mathbf{u})]^{d} d \mathbf{u}=\int_{S^{2}}\left[f\left(R^{-1} \mathbf{u}\right)\right]^{d} d \mathbf{u}
$$

Although it is an interesting RIF, $I^{d}[f]$ represents only a partial representation of $f$. In order to overcome this limitation we can define a new set of RIF from $I^{d}[f]$. In particular, we observe that we can write $I^{d}[f]$ as

$$
\begin{aligned}
I^{d}[f] & =\sum_{l_{1}=0}^{\infty} \cdots \sum_{l_{d}=0}^{\infty} \int_{S^{2}} \prod_{i=1}^{d}\left[\sum_{m_{i}=-l_{i}}^{l_{i}} c_{l_{i} m_{i}} Y_{l_{i}}^{m_{i}}(\mathbf{u})\right] d \mathbf{u} \\
& =\sum_{l_{1}=0}^{\infty} \cdots \sum_{l_{d}=0}^{\infty} \int_{S^{2}} \sum_{m_{1}=-l_{1}}^{l_{1}} \cdots \sum_{m_{d}=-l_{d}}^{l_{d}} c_{l_{1} m_{1}} \cdots c_{l_{d} m_{d}} Y_{l_{1}}^{m_{1}}(\mathbf{u}) \cdots Y_{l_{d}}^{m_{d}}(\mathbf{u}) d \mathbf{u} \\
& =\sum_{l_{1}=0}^{\infty} \cdots \sum_{l_{d}=0}^{\infty} \sum_{m_{1}=-l_{1}}^{l_{1}} \cdots \sum_{m_{d}=-l_{d}}^{l_{d}} c_{l_{1} m_{1}} \cdots c_{l_{d} m_{d}} \int_{S^{2}} Y_{l_{1}}^{m_{1}}(\mathbf{u}) \cdots Y_{l_{d}}^{m_{d}}(\mathbf{u}) d \mathbf{u} \\
& =\sum_{l_{1}=0}^{\infty} \cdots \sum_{l_{d}=0}^{\infty} I_{l_{1} \ldots l_{d}}^{d}[f]=\sum_{1} I_{1}^{d}[f]
\end{aligned}
$$

with $\mathbf{l}=\left[l_{1} \ldots l_{d}\right] . I_{\mathbf{l}}^{d}[f]$ can be evaluated as

$$
I_{l_{1} \ldots l_{d}}^{d}[f]=\sum_{m_{1}=-l_{1}}^{l_{1}} \cdots \sum_{m_{d}=-l_{d}}^{l_{d}} c_{l_{1} m_{1}} \cdots c_{l_{d} m_{d}} G\left(l_{1}, m_{1}|\cdots| l_{d}, m_{d}\right)
$$

where $G\left(l_{1}, m_{1}|\cdots| l_{d}, m_{d}\right)$ represents the generalized Gaunt coefficient calculated as the integral of the product of $d \mathrm{SH}$ of the same argument [7]. We can also view $I_{1}^{d}$ as a polynomial of degree $d$ of the SH coefficients $c_{l m}$.

Appendix A provides a proof that Equation (12) generates rotation invariant features of the spherical function $f(\mathbf{u})$ given the set of SH indices $\mathbf{l}$. The function $I_{1}^{d}$ shares some of the properties of the Gaunt coefficients, in particular it is zero for most combination of $\mathbf{l}$ and it is invariant to the permutations of the indices. In this work, we will refer to the set of all the non-zero invariants of maximal SH order $l_{\max }$ and degree $d$ as $\mathbb{I}_{l_{\max }}^{d}$.

\subsection{Example 1: degree 2 invariant-polynomials}

In the case of polynomials of degree $d=2$ and considering a maximal SH order $l_{\max }=2, I_{1}^{d}$ corresponds to

$$
I_{l_{1}, l_{2}}^{2}[f]=\sum_{m_{1}=-l_{1}}^{l_{1}} \sum_{m_{2}=-l_{2}}^{l_{2}} c_{l_{1} m_{1}} c_{l_{2} m_{2}} G\left(l_{1}, m_{1} \mid l_{2}, m_{2}\right)
$$




\begin{tabular}{c|c|c|c|c|c} 
& $d=1$ & $d=2$ & $d=3$ & $d=4$ & $d=5$ \\
\hline$l_{\max }=2$ & $1(\mathbf{1})$ & $2(\mathbf{2})$ & $3\left(\mathbf{3}^{*}\right)$ & $4\left(\mathbf{3}^{*}\right)$ & $5\left(\mathbf{3}^{*}\right)$ \\
$l_{\max }=4$ & $1(\mathbf{1})$ & $5(\mathbf{3})$ & $7(\mathbf{7})$ & $12(\mathbf{1 1})$ & $18\left(\mathbf{1 2}^{*}\right)$ \\
$l_{\max }=6$ & $1(\mathbf{1})$ & $7(\mathbf{4})$ & $13(\mathbf{1 3})$ & $28\left(\mathbf{2 5}^{*}\right)$ & $49\left(\mathbf{2 5}^{*}\right)$ \\
\hline
\end{tabular}

Table 1. Number of RIF for symmetric (even) SH basis given the maximum value of $\mathbf{l}$ and the degree. In bold we report the number of algebraically independent invariants, with an asterisk if the number corresponds to the maximum theoretical number of invariants.

with $\mathbf{l}=[(0,0),(0,1), \cdots(2,2)]$ considering only the sorted couple in ascending order of $\left(l_{1}, l_{2}\right)$ because of the symmetry property of the Gaunt coefficients. $G\left(l_{1}, m_{1} \mid l_{2}, m_{2}\right)$ represents the integral of two $\mathrm{SH}$ which is equal to $\delta_{l_{1}, m_{1}}^{l_{2}, m_{2}}$. This means that the invariant will be non-zero only when $l_{1}=l_{2}$ and $m_{1}=m_{2}$. We can thus rewrite the degree two invariants as

$$
I_{l}^{2}[f]=\sum_{m=-l}^{l}\left[c_{l, m}\right]^{2}
$$

which corresponds to the power spectrum of the $\mathrm{SH}$ basis. The power spectrum is one of the most widely used invariants in pattern recognition application and has been recently introduced in diffusion MRI for tissue microstructure estimation $[12]$.

\subsection{Example 2: degree 3 invariant-polynomials}

The invariants of degree 3 can be calculated as

$$
I_{l_{1}, l_{2}, l_{3}}^{3}[f]=\sum_{m_{1}=-l_{1}}^{l_{1}} \sum_{m_{2}=-l_{2}}^{l_{2}} \sum_{m_{3}=-l_{3}}^{l_{3}} c_{l_{1} m_{1}} c_{l_{2} m_{2}} c_{l_{3} m_{3}} G\left(l_{1}, m_{1}\left|l_{2}, m_{2}\right| l_{3}, m_{3}\right)
$$

It is possible to show that $I_{l_{1}, l_{2}, l_{3}}^{3}[f]$ are actually equivalent to the bispectrum invariants proposed in [10], [9], and [11] (results not shown due to space limitation). The main difference with respect to the bispectrum is that in this case we have the Gaunt coefficients obtained from the integral of three SH instead of the Clebsch-Gordan coefficients.

It is possible to show that both $I_{l_{1}, l_{2}}^{2}$ and $I_{l_{1}, l_{2}}^{3}$ are not only invariants but they also form a set of algebraically independent invariants. The next subsection will provide the formal definition of algebraically independence and how to apply it in order to verify the independence of any set of $\mathbb{I}_{l_{\max }}^{d}$.

\subsection{Algebraically independence}

Definition 1. A set of $m$ polynomials $P_{1}, \ldots, P_{m} \in R\left[X_{1}, \ldots, X_{n}\right]$ are called algebraically independent over the field $R$ if 


\begin{tabular}{l|c|c|c|c} 
& $d=1$ & $d=2$ & $d=3$ & $d=4$ \\
\hline$l_{\max }=2$ & $1(\mathbf{1})$ & $3(\mathbf{3})$ & $5(\mathbf{5})$ & $8\left(\mathbf{6}^{*}\right)$ \\
$l_{\max }=3$ & $1(\mathbf{1})$ & $4(\mathbf{4})$ & $8(\mathbf{8})$ & $17\left(\mathbf{1 3}^{*}\right)$ \\
$l_{\max }=4$ & $1(\mathbf{1})$ & $5(\mathbf{5})$ & $14(\mathbf{1 4})$ & $33\left(\mathbf{2 2}^{*}\right)$ \\
$l_{\max }=5$ & $1(\mathbf{1})$ & $6(\mathbf{6})$ & $20(\mathbf{2 0})$ & $57\left(\mathbf{3 3}^{*}\right)$ \\
$l_{\max }=6$ & $1(\mathbf{1})$ & $7(\mathbf{7})$ & $30(\mathbf{3 0})$ & $94\left(\mathbf{4 6}^{*}\right)$ \\
\hline
\end{tabular}

Table 2. Number of RIF given the maximum value of $\mathbf{l}$ and the degree. In bold we report the number of algebraically independent invariants, with an asterisk if the number corresponds to the maximum theoretical number of invariants.

$$
\forall Q \in R\left[Y_{1}, \ldots, Y_{m}\right], \quad Q\left(P_{1}, \ldots, P_{m}\right)=0 \Longleftrightarrow Q=0
$$

Example Given $P_{1}=2 x^{2}-4 x y+2 y^{2}$ and $P_{2}=x-y$, the set $\left[P_{1}, P_{2}\right]$ is not algebraically independent. In fact, considering $Q=2 P_{2}^{2}-P_{1}$ it leads to: $2(x-y)^{2}-\left(2 x^{2}-4 x y+2 y^{2}\right)=0$.

An efficient way for verifying whether the invariants $\mathbb{I}_{l_{\max }}^{d}$ are algebraically independent is the Jacobian theorem proposed by [5]:

Theorem 1 The polynomials $P_{1}, \ldots, P_{m} \in R\left[X_{1}, \ldots, X_{n}\right]$ with $m \leq n$ are algebraically independent iff the Jacobian matrix $\left(\partial P_{i} / \partial X_{j}\right)_{1 \leq i \leq m, 1 \leq j \leq n}$ has full rank.

In our case, the Jacobian matrix corresponds to the partial derivatives of our invariants $I_{1}^{d}$ with respect to the SH coefficients $c_{l m}$. The testing for full-rankness is performed taking advantage of the Schwartz-Zippel polynomial identity testing lemma [16][20] which states that given a random vector of coefficients c, the Jacobian rank corresponds to the rank of $\left(\partial P_{i} / \partial X_{j}\right)_{X=\mathbf{c}}$ with a probability that is proportional to the probability of extracting $\mathbf{c}$ randomly. In this work, we consider 100 random instances of $c_{l m}$ in order to assess the independence of $I_{1}^{d}$ with sufficient accuracy.

This approach has already been used for the same purpose by [2], where the authors also theorized that the maximal number of independent coefficients is equal to the maximal number of $\mathrm{SH}$ coefficients used, $n_{c}$, minus three, the degrees of freedom of the rotation. Table 1 show the number of invariants calculated for the symmetric SH basis [4]. In bold we highlight the number of algebraically independent invariants and we also add an asterisk if this number corresponds to $n_{c}-3$. For the complete SH basis we observed that using $d=4$ we are able to obtain all the invariants up to $l_{\max }=6$, while for the symmetric SH basis our technique require $d=5$ in order to obtain the $12^{\text {th }}$ invariant for $l_{\max }=4$. Caruyer and Verma [2] were able to obtain all 12 invariants for $l_{\max }=4$ at $d=4$ using a different technique, and we are also able to obtain the complete set of invariants at $d=4$ if we include also the odd terms of the basis (see Table 2 ). It is possible that with our technique the $12^{\text {th }}$ invariant can be expressed as a combination of two mixed even-odd invariants and appears as a function of purely even coefficients only at $d=5$. 
Note that with our technique all the invariants at a certain degree include also all the invariants of lower degree (i.e. $I_{2,2}^{2}$ represents the same invariant as $I_{0,0,2,2}^{4}$ ). Therefore, in order to calculate all the algebraically independent invariants it is sufficient to consider only the degree $d$ that provides the maximal number of invariants, without having to compute all the invariants at lower degree. For example, taking Table 1 first row, we can observe that we have two invariants at $d=2$ of which one is a new invariant and the other is the same invariant that appears at $d=1$. In practice, in order to obtain the number of new invariants appearing only at degree $d$ we have to subtract the numbers of invariants of degree $d-1$.

\section{Materials and methods}

Synthetic fODF dataset: We created a synthetic fODF dataset consisting of crossings of two delta functions on the sphere.

We can obtain the SH coefficients of two delta functions with equal volume fraction centered around $\mathbf{v}_{1}$ and $\mathbf{v}_{2}$, respectively, as

$$
c_{l, m}^{\text {crossing }}=\frac{1}{2} Y_{l}^{m}\left(\mathbf{v}_{1}\right)+\frac{1}{2} Y_{l}^{m}\left(\mathbf{v}_{2}\right)
$$

In this dataset, we will use the cosine of the crossing angle, $\mathbf{v}_{1} \cdot \mathbf{v}_{2}$, as the reference value for the invariants. Since the maximum absolute value of our RIF is obtained when the considered fODF is a delta function $(O D=0$ or $\mathbf{v}_{1} \cdot \mathbf{v}_{2}= \pm 1$ ) we can use this value as a normalization factor for our invariants. In this work we will call the normalized fODF invariants $\hat{I}_{1}^{d}[\rho]$ to distinguish them from the unnormalized version.

Human Connectome Project (HCP) dataset: In order to test our RIF in-vivo we considered one subject of the HCP diffusion MRI dataset [17]. The HCP acquisition scheme presents $18 b$-value $0 \mathrm{~s} / \mathrm{mm}^{2}$ volumes, and 90 volumes at $b$ value 1000,2000 , and $3000 \mathrm{~s} / \mathrm{mm}^{2}$, respectively. All the three shell were acquired using an unique gradient direction. HCP pulse separation time was set at $\Delta=$ $43.1 \mathrm{~ms}$ and pulse width at $\delta=10.6 \mathrm{~ms}$. Each MRI volume consists of $145 \times 174 \times$ 145 voxels with a resolution of $1.25 \times 1.25 \times 1.25 \mathrm{~mm}^{3}$.

\section{Results}

Figure 1 shows the proposed algebraically independent RIF given a maximum $\mathrm{SH}$ order $l_{\max }=4$ for the synthetic fODF dataset, before (left) and after (right) the normalization by the maximum theoretical value. This dataset was generated by taking 1000 fODF directions $\mathbf{v}_{1}$ and $\mathbf{v}_{2}$ randomly, and the relative invariants

was ordered according to the value of $\mathbf{v}_{1} \cdot \mathbf{v}_{2}$. Because of the randomness of the orientation of the crossings, the smoothness of the curves itself can be view as an empirical proof of the rotation invariance property of our RIF. In fact, 

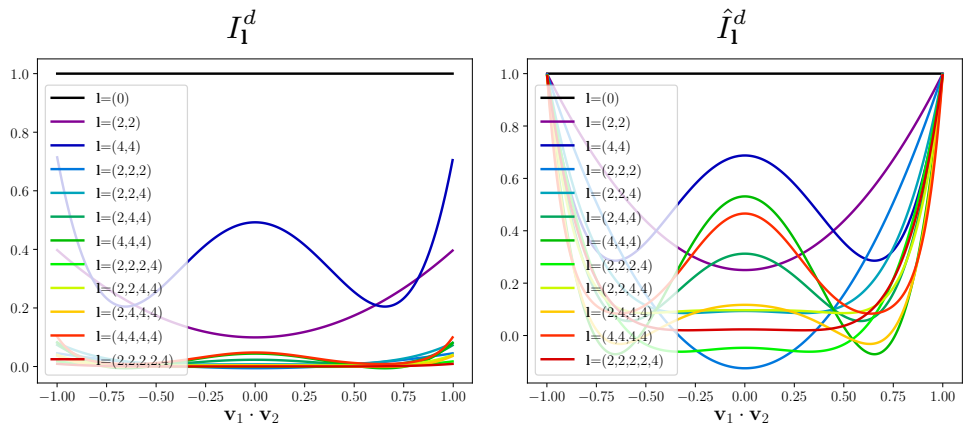

Fig. 1. Unnormalized (left) and normalized (right) visual representation of the 12 algebraically independent invariants for $l_{\max }=4$ with respect to different crossing angles.

\begin{tabular}{l|c|c|c|c} 
& $0^{\circ}$ & $30^{\circ}$ & $60^{\circ}$ & $90^{\circ}$ \\
\hline $\mathbf{l}=(0)$ & $1.00 / 0.000$ & $1.00 / 0.000$ & $1.00 / 0.000$ & $1.00 / 0.000$ \\
$\mathbf{l}=(2,2)$ & $0.89 / 0.012$ & $0.75 / 0.012$ & $0.39 / 0.009$ & $0.23 / 0.007$ \\
$\mathbf{l}=(4,4)$ & $0.67 / 0.021$ & $0.38 / 0.014$ & $0.23 / 0.012$ & $0.46 / 0.016$ \\
$\mathbf{l}=(2,2,2)$ & $0.85 / 0.017$ & $0.64 / 0.016$ & $0.13 / 0.008$ & $-0.11 / 0.005$ \\
$\mathbf{l}=(2,2,4)$ & $0.73 / 0.017$ & $0.45 / 0.012$ & $0.09 / 0.006$ & $0.07 / 0.003$ \\
$\mathbf{l}=(2,4,4)$ & $0.63 / 0.021$ & $0.30 / 0.012$ & $0.04 / 0.004$ & $0.21 / 0.010$ \\
$\mathbf{l}=(4,4,4)$ & $0.54 / 0.026$ & $0.19 / 0.012$ & $0.02 / 0.009$ & $0.28 / 0.018$ \\
$\mathbf{l}=(2,2,2,4)$ & $0.69 / 0.019$ & $0.38 / 0.013$ & $-0.02 / 0.003$ & $-0.03 / 0.002$ \\
$\mathbf{l}=(2,2,4,4)$ & $0.60 / 0.022$ & $0.28 / 0.012$ & $0.05 / 0.004$ & $0.06 / 0.003$ \\
$\mathbf{l}=(2,4,4,4)$ & $0.51 / 0.025$ & $0.17 / 0.010$ & $-0.00 / 0.002$ & $0.06 / 0.003$ \\
$\mathbf{l}=(4,4,4,4)$ & $0.45 / 0.028$ & $0.15 / 0.011$ & $0.05 / 0.005$ & $0.21 / 0.014$ \\
$\mathbf{l}=(2,2,2,2,4)$ & $0.66 / 0.022$ & $0.34 / 0.014$ & $0.03 / 0.003$ & $0.02 / 0.001$ \\
\hline
\end{tabular}

Table 3. Mean and standard deviation of the 12 algebraically independent invariants for $l_{\max }=4$ with respect to different crossing angles.

two adjacent points in the graph had been generated by crossings oriented, up to a rotation, in two completely different directions. If any of the $I_{1}^{d}$ was not invariant its curve would have appear as a broken line in the graph. We also test the robustness of the invariants with respect to noise. We simulate diffusion signal arising from crossing fibers at 0, 30, 60, and 90 degrees. We add Rician noise with a signal to noise ratio equal to 30 , generating 100 noise realization per crossing angle. The fODF have been calculated using constrained spherical deconvolution algorithm with SH order equal to 4 . Table 3 reports the mean and standard deviation of the twelve algebraically independent invariants. In general, our invariants result to be stable with values of standard deviation fifteen times lower than the mean in the worst case. Invariants including higher harmonics terms seem to present higher standard deviation $(\mathrm{e} . \mathrm{g} \mathbf{l}=(2,2)$ is more stable than $\mathbf{l}=(4,4))$. This is probably due to the fact that higher harmonics tend to model signal noise more with respect to the lower ones that are too smooth to capture rapid signal changes. 
In order to test the invariants in-vivo we consider one subject of the HCP dataset. The main advantage of this dataset is the high spatial resolution which enables us to observe the fine changes of our RIF in the white matter. From the diffusion signal, a voxel-dependent response function was obtained using the multi-compartment model presented in [8]. Each response function has been used for calculating the fODF with constrained spherical deconvolution $\left(l_{\max }=\right.$ $4)$ as in [21]. Figure 2 shows the 12 algebraically independent invariants after normalization for a coronal slice of the HCP subject. As for the synthetic data, the first invariant $\mathbf{l}=(0)$ is constant because the first $\mathrm{SH}$ coefficient $c_{00}$ is always $1 / \sqrt{4 \pi}$ in order to have the integral of the fODF equal to 1 [12]. The average values of the invariants generally decrease with the increase of each $l_{i}$ and with the degree of the polynomials. For example, $\mathbf{l}=(2,2)$ has an average of 0.12 while $\mathbf{l}=(2,2,2,2,4)$ has an average of only 0.0009 in white matter. Conversely, the contrast between white matter and gray matter generally increases with the degree. A similar trend can be observed in the contrast between single bundles and crossings in white matter. Some of our RIF present negative values in certain areas of the brain, in particular in fiber crossing regions. In our simulations we observed that negative values for certain invariants are associated with a characteristic crossing angle (e.g 90 degrees crossing for $\mathbf{l}=(2,2,2))$. However, our synthetic data is formed only of 2-fiber crossings, whereas crossings of three or even more fibers are common in the human brain. Therefore, a direct mapping of the value of the RIF to the fiber geometry is not trivial and will require more advanced mathematical tools.

\section{Discussion and Conclusion}

In this work, we proposed a new framework for generating algebraically independent rotation invariant features for the diffusion MRI signal. One of the main results of our technique is that, conversely with respect to other works, we were able to provide a general closed-form for our invariants. The core mathematical tool of our formulation are the Gaunt coefficients originating from the integral of multiple SH. Gaunt coefficients also appear in the invariants for diffusion MRI proposed in [15], although using a completely different approach and without testing the resulting invariants for completeness or independence.

Rotation invariant features are not only a subject of interest in diffusion MRI but also in several other fields including computer vision and pattern recognition [9][10]. We believe that our framework will be advantageous for non-diffusion applications given the simplicity of the mathematical formulation of our RIF. Our mathematical formulation makes them very fast to compute even for large SH order compared to other techniques.

Recent works [12][14] show the importance of rotation invariant features such as the power spectrum for estimating tissue microstructural properties. We will focus our future works in trying to establish whether including the complete set of invariants will result in a more precise estimation of brain microstructure. 

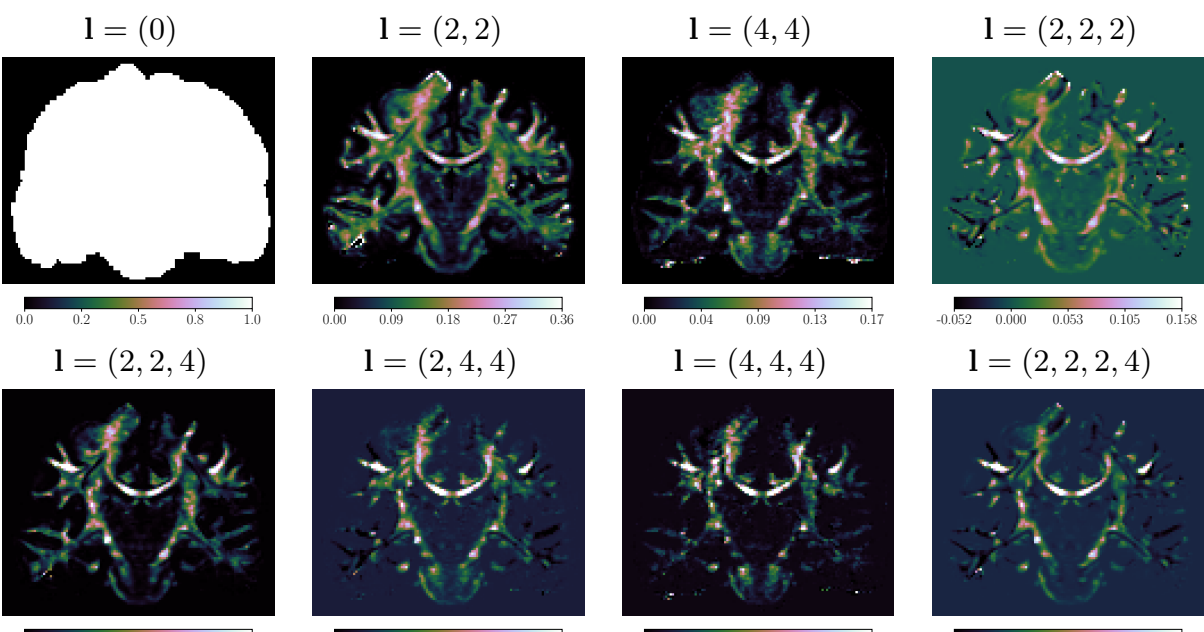

$\mathbf{l}=(4,4,4)$
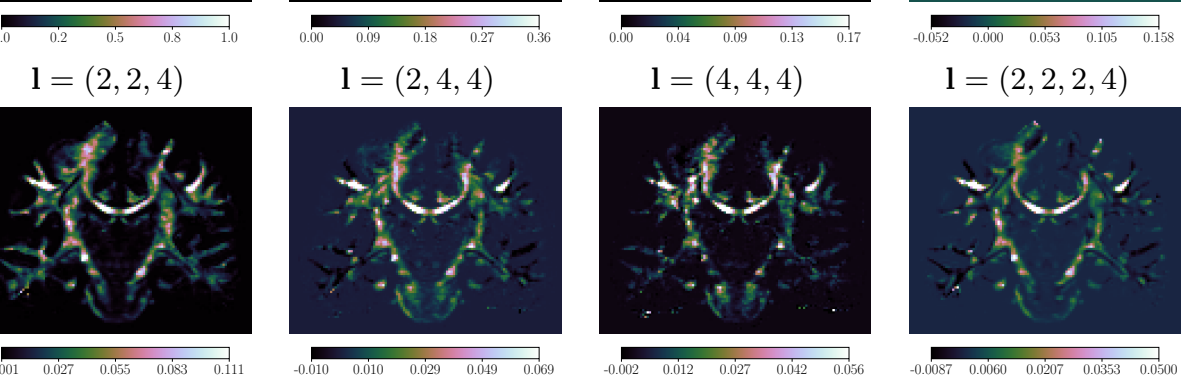

$\mathbf{l}=(2,2,2,4)$

$\mathbf{l}=(2,2,4,4)$

$$
\mathbf{l}=(2,4,4,4)
$$

$\mathbf{l}=(4,4,4,4)$
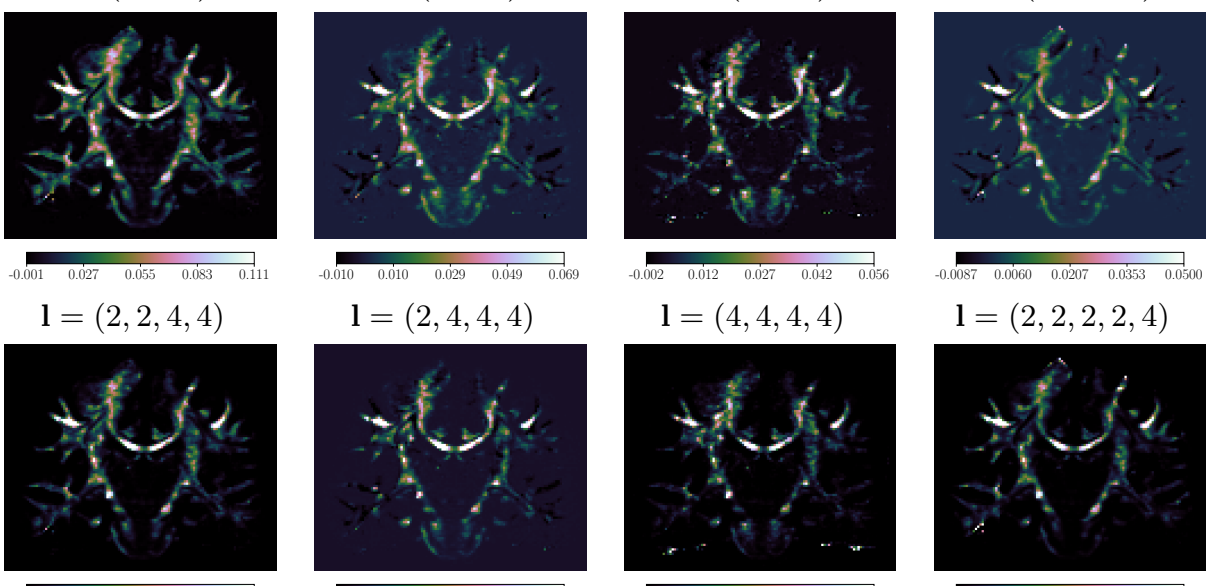

$\mathbf{l}=(2,2,2,2,4)$

$\begin{array}{lllll}0.0000 & 0.0106 & 0.0212 & 0.0318 & 0.0424\end{array}$

$\begin{array}{llllll}-0.0025 & 0.0047 & 0.0118 & 0.0190 & 0.0261\end{array}$

$\begin{array}{lllll}0.0000 & 0.0072 & 0.0145 & 0.0217 & 0.0290\end{array}$

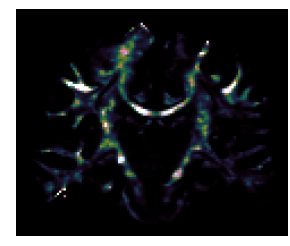

$0.00004 \quad 0.008190 .016420 .024660 .03289$

Fig. 2. All the 12 algebraically independent invariants calculated on the fODF of a coronal slice of one of the HCP subjects.

\section{Acknowledgment}

This work has received funding from the European Research Council (ERC) under the European Union's Horizon 2020 research and innovation program (ERC Advanced Grant agreement No 694665 : CoBCoM - Computational Brain Connectivity Mapping).

Data were provided by the Human Connectome Project, WU-Minn Consortium (Principal Investigators: David Van Essen and Kamil Ugurbil; 1U54MH091657) funded by the 16 NIH Institutes and Centers that support the NIH Blueprint for Neuroscience Research; and by the McDonnell Center for Systems Neuroscience at Washington University. 


\section{A Relation between signal SH coefficients and fODF SH coefficients}

$I_{1}^{d}[f]$ is rotation invariant iff $I_{1}^{d}[f]=I_{1}^{d}[R f]=I_{1}^{d}[h]$. Given the rotated SH expansion $h(\mathbf{u})$ we can calculate $I_{1}^{d}[h]$ as

$$
\begin{aligned}
I_{l_{1} \ldots l_{d}}^{d}[h] & =\int_{S^{2}} \prod_{i=1}^{d}\left[\sum_{m_{i}^{\prime}=-l_{i}}^{l_{i}} g_{l_{i} m_{i}^{\prime}} Y_{l_{i}}^{m_{i}^{\prime}}(\mathbf{u})\right] d \mathbf{u} \\
& =\int_{S^{2}} \prod_{i=1}^{d}\left[\sum_{m_{i}^{\prime}=-l_{i}}^{l_{i}} \sum_{m_{i}=-l_{i}}^{l_{i}} c_{l_{i} m_{i}} D_{m_{i}^{\prime}, m_{i}}^{l_{i}}(R) Y_{l_{i}}^{m_{i}^{\prime}}(\mathbf{u})\right] d \mathbf{u} \\
& =\int_{S^{2}} \prod_{i=1}^{d}\left[\sum_{m_{i}=-l_{i}}^{l_{i}} c_{l_{i} m_{i}} \sum_{m_{i}^{\prime}=-l_{i}}^{l_{i}} D_{m_{i}^{\prime}, m_{i}}^{l_{i}}(R) Y_{l_{i}}^{m_{i}^{\prime}}(\mathbf{u})\right] d \mathbf{u} \\
& =\int_{S^{2}} \prod_{i=1}^{d}\left[\sum_{m_{i}=-l_{i}}^{l_{i}} c_{l_{i} m_{i}} Y_{l}^{m}\left(R^{-1} \mathbf{u}\right)\right] d \mathbf{u} \\
& =\int_{S^{2}} \sum_{m_{1}=-l_{1}}^{l_{1}} \ldots \sum_{m_{d}=-l_{d}}^{l_{d}} c_{l_{1} m_{1}} \cdots c_{l_{d} m_{d}} Y_{l_{1}}^{m_{1}}\left(R^{-1} \mathbf{u}\right) \cdots Y_{l_{d}}^{m_{d}}\left(R^{-1} \mathbf{u}\right) d \mathbf{u} \\
& =\sum_{m_{1}=-l_{1}}^{l_{1}} \ldots \sum_{m_{d}=-l_{d}}^{l_{d}} c_{l_{1} m_{1}} \cdots c_{l_{d} m_{d}} \int_{S^{2}} Y_{l_{1}}^{m_{1}}\left(R^{-1} \mathbf{u}\right) \cdots Y_{l_{d}}^{m_{d}}\left(R^{-1} \mathbf{u}\right) d \mathbf{u} \\
& =\sum_{m_{1}=-l_{1}}^{l_{1}} \ldots \sum_{m_{d}=-l_{d}}^{l_{d}} c_{l_{1} m_{1}} \cdots c_{l_{d} m_{d}} G\left(l_{1}, m_{1}|\cdots| l_{d}, m_{d}\right) \\
& =I_{l_{1} \ldots l_{d}}^{d}[f]
\end{aligned}
$$

which proves the invariance property $I_{1}^{d}[f]=I_{1}^{d}[R f]$.

\section{References}

1. Bloy, L., Verma, R.: Demons registration of high angular resolution diffusion images. In: 2010 IEEE International Symposium on Biomedical Imaging: From Nano to Macro. pp. 1013-1016 (April 2010)

2. Caruyer, E., Verma, R.: On facilitating the use of hardi in population studies by creating rotation-invariant markers. Medical image analysis 20(1), 87-96 (2015)

3. Dell'Acqua, F., Scifo, P., Rizzo, G., Catani, M., Simmons, A., Scotti, G., Fazio, F.: A modified damped richardson-lucy algorithm to reduce isotropic background effects in spherical deconvolution. Neuroimage 49(2), 1446-1458 (2010)

4. Descoteaux, M., Angelino, E., Fitzgibbons, S., Deriche, R.: Regularized, fast, and robust analytical q-ball imaging. Magnetic Resonance in Medicine 58(3), 497-510 (2007) 
5. Ehrenborg, R., Rota, G.C.: Apolarity and canonical forms for homogeneous polynomials. European Journal of Combinatorics 14(3), 157-181 (1993)

6. Ghosh, A., Papadopoulo, T., Deriche, R.: Generalized invariants of a 4 th order tensor: Building blocks for new biomarkers in dmri. In: Proceedings of the Computation Diffusion MRI Workshop at the MICCAI Conference (2012)

7. Homeier, H.H., Steinborn, E.: Some properties of the coupling coefficients of real spherical harmonics and their relation to gaunt coefficients. Journal of Molecular Structure: THEOCHEM 368, 31 - 37 (1996), proceedings of the Second Electronic Computational Chemistry Conference

8. Kaden, E., Kelm, N.D., Carson, R.P., Does, M.D., Alexander, D.C.: Multicompartment microscopic diffusion imaging. NeuroImage 139, 346 - 359 (2016)

9. Kakarala, R., Mao, D.: A theory of phase-sensitive rotation invariance with spherical harmonic and moment-based representations. In: 2010 IEEE Computer Society Conference on Computer Vision and Pattern Recognition. pp. 105-112 (June 2010)

10. Kondor, R.: A novel set of rotationally and translationally invariant features for images based on the non-commutative bispectrum. arXiv preprint cs/0701127 (2007)

11. Negrinho, R.M.P., Aguiar, P.M.Q.: Shape representation via elementary symmetric polynomials: A complete invariant inspired by the bispectrum. In: 2013 IEEE International Conference on Image Processing. pp. 3518-3522 (Sept 2013)

12. Novikov, D.S., Veraart, J., Jelescu, I.O., Fieremans, E.: Rotationally-invariant mapping of scalar and orientational metrics of neuronal microstructure with diffusion mri. NeuroImage 174, 518 - 538 (2018)

13. Papadopoulo, T., Ghosh, A., Deriche, R.: Complete set of invariants of a 4th order tensor: The 12 tasks of hardi from ternary quartics. In: Golland, P., Hata, N., Barillot, C., Hornegger, J., Howe, R. (eds.) Medical Image Computing and Computer-Assisted Intervention - MICCAI 2014. pp. 233-240. Springer International Publishing, Cham (2014)

14. Reisert, M., Kellner, E., Dhital, B., Hennig, J., Kiselev, V.G.: Disentangling micro from mesostructure by diffusion mri: A bayesian approach. NeuroImage pp. (2016)

15. Schwab, E., Çetingül, H.E., Afsari, B., Yassa, M.A., Vidal, R.: Rotation invariant features for hardi. In: Gee, J.C., Joshi, S., Pohl, K.M., Wells, W.M., Zöllei, L. (eds.) Information Processing in Medical Imaging. pp. 705-717. Springer Berlin Heidelberg, Berlin, Heidelberg (2013)

16. Schwartz, J.T.: Fast probabilistic algorithms for verification of polynomial identities. J. ACM 27(4), 701-717 (Oct 1980)

17. Sotiropoulos, S.N., Jbabdi, S., Xu, J., Andersson, J.L., Moeller, S., Auerbach, E.J., Glasser, M.F., Hernandez, M., Sapiro, G., Jenkinson, M., et al.: Advances in diffusion MRI acquisition and processing in the human connectome project. Neuroimage 80, 125-143 (2013)

18. Tournier, J.D., Calamante, F., Connelly, A.: Robust determination of the fibre orientation distribution in diffusion mri: non-negativity constrained super-resolved spherical deconvolution. NeuroImage 35(4), 1459-1472 (2007)

19. Tuch, D.S.: Q-ball imaging. Magnetic Resonance in Medicine 52(6), 1358-1372

20. Zippel, R.: Probabilistic algorithms for sparse polynomials. In: Ng, E.W. (ed.) Symbolic and Algebraic Computation. pp. 216-226. Springer Berlin Heidelberg, Berlin, Heidelberg (1979)

21. Zucchelli, M., Descoteaux, M., Menegaz, G.: A generalized smt-based framework for diffusion mri microstructural model estimation. In: Computational Diffusion MRI, pp. 51-63. Springer (2018) 